\title{
The Study of PSi \& PSo Algorithm for Reducing Power of the Mobile Communication Network
}

\author{
Jongsin $\mathrm{Kim}^{*}$, , Yonggil $\mathrm{Choi}^{2}$, Younghoon $\mathrm{Oh}^{2}$ \\ ${ }^{1}$ Department of IT Policy and Management, Soongsil University, 06978, South Korea
}

${ }^{2}$ SK Telecom, Western Infra Engineering Team, 62355, South Korea

\begin{tabular}{l} 
A R T I C L E I N F O \\
\hline Article history: \\
Received: 09 April 2019 \\
Accepted: 26 June, 2019 \\
Online: 11 July, 2019 \\
\hline Keywords: \\
Power Saving \\
Energy Saving \\
4G/5G \\
LTE \\
Algorithm
\end{tabular}

\begin{abstract}
A B S T R A C T
Recently, the trend of mobile communication network in Korea has been dramatically increasing the number of operating equipment for managing the explosion of wireless data traffic in $4 G$ LTE network. This phenomenon has caused an increase in the operating cost to the mobile telecommunication service provider. Especially, the study about the reduction of the operating cost through the reduction of the power consumption in the power consumption area and the efficient operation method are under study. Through these studies, we consider the method to maintain the best service quality of mobile communication subscriber while minimizing the power consumption of LTE equipment, find out PSi \& PSo algorithm that can realize this and verify it in commercial network. Applying this algorithm to a commercial network will save both power consumption and service experience of mobile subscribers to the best level, and it will be more effective in the $5 G$ era that has already begun.
\end{abstract}

\section{Introduction}

This paper is an extension of work originally presented in 2018 International Conference on Information and Communication Technology Convergence (ICTC) [1].

The world has already begun to enter the $5 \mathrm{G}$ world, and the first $5 \mathrm{G}$ signal in Korea has started to be transmitted. There are also many killer services available to satisfy customers' needs. This means that a lot of people are using smartphones and are getting into daily life. In the past, the use patterns of voice-based usage changed with data, and the network that accommodates customers changed quickly. As the LTE network matured, the frequency bandwidth and the number of equipment services are continuously increasing to accommodate the increasing subscriber traffic [2].

This provides a pleasant environment for various services and accommodates a growing number of subscribers, but the cost of operating of equipment has increased. SK Telecom's electricity costs account for about $30 \%$ of the total cost of operating a network, exceeding 30\% in 2017, which is still rising. In 2019, electricity costs are expected to account for $35 \%$ of total operating costs.

This increase in network operating costs is bringing considerable stress to mobile telecom operators, and it is also

\footnotetext{
*Jongsin Kim, +8210-3768-6309, Email: kjs3838@sk.com
}

evolving to a level that significantly affects corporate EBITDA [3]. As a result, each company has a great interest in reducing the cost of network operation, especially in the field of electric power. In previous mobile communication areas, there has been much interest in battery efficiency aspects such as SoC (System on Chip) upgrade [4] and C-DRX(Connected Mode Discontinuous Reception) [5]. Recently, attention has been focused on the reduction of power consumption of network equipment itself.

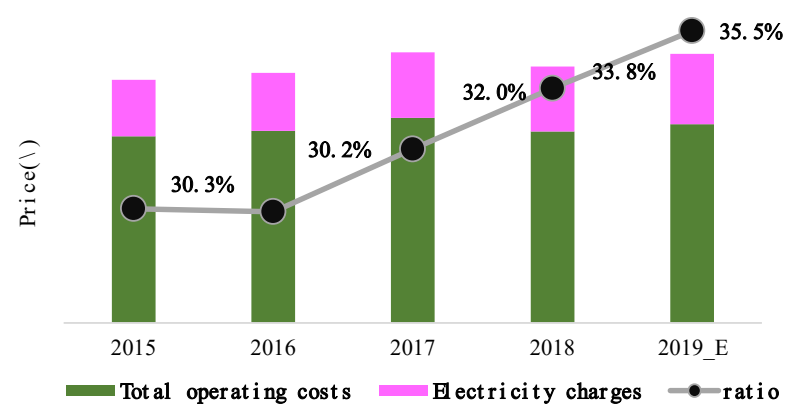

Figure 1: Electricity charges and ratio in operating costs

We used the power saving function of SK Telecom's NOKIA equipment to investigate how to reduce the power consumption of the network equipment itself. Through the derivation of optimal values of PSi and PSo, which are control variables for triggering 
power saving to be explained later in detail, Consumption is decreasing. The optimal PSi and PSo values mean the point where the quality of the mobile communication subscriber can be maximized while minimizing the power consumption.

In this paper, we propose an operation algorithm that can reduce the power consumption of wireless network service equipment while providing optimal service without inconvenience to mobile communication subscribers. In this process, SK Telecom's commercial network is divided into several cases, and results are derived. The power saving function of the wireless network service equipment and the algorithms discussed in this study are used to reduce the actual power consumption.

\section{Basic direction of power saving of LTE wireless network}

Table 1: Number of subscribers per 10Mbps

\begin{tabular}{|c|c|}
\hline Freq. Combination & Number of eligible subscribers \\
\hline $800 \mathrm{M}$ & 7.5 \\
\hline $800 \mathrm{M}+1.8 \mathrm{G}$ & 22.5 \\
\hline $800 \mathrm{M}+1.8 \mathrm{G}+2.1 \mathrm{G}$ & 30.0 \\
\hline $800 \mathrm{M}+1.8 \mathrm{G}+2.1 \mathrm{G}+2.6 \mathrm{G}$ & 52.5 \\
\hline
\end{tabular}

Table 1 shows the number of subscribers per frequency configuration operated by SK Telecom. As shown in the table, the number of subscribers that can be accommodated for each frequency combination is linear. (However, in LTE network, the service required for voice, messaging, video, and FTP is different according to service type, but it is calculated based on 10Mbps speed for convenience.) In order to service many subscribers, more frequency and bandwidth are needed. On the contrary, it is not necessary to provide the frequency and bandwidth of mobile communication subscribers in every place. This is because the number of subscribers and traffic vary with time. In other words, the frequency and bandwidth need to be tailored to the region's maximum traffic, but not all-time full-band service. Taking operational efficiency in accordance with the traffic volume and characteristics of subscribers is a basic direction for power saving in LTE networks.

In order to reduce power consumption in LTE mobile communication, there is a method to utilize a SON (Self Organizing Network) [6] implemented by a mobile communication provider. As mentioned above, this study utilized the power saving function provided by NOKIA equipment.(Other vendors, however, offer similar functionality.) The basic concept of power saving is that when the same area is served by several frequencies, the subscriber traffic of the service area is judged as a combined load, and when this value is low, the power consumption is reduced by turning off the output of some frequencies.

As can be seen in Figure 2, when several frequencies are serviced in the same area, the number of subscribers and traffic varies with time, and thus the number of frequencies required varies. Typically, however, mobile operators have been providing full-band services in their area, which means that unnecessary power consumption is still happening. One thing to notice is that traffic loads do not change over time in all locations. It should be noted that the traffic varies according to the characteristics of the area and the subscriber, and thus the service quality of the mobile communication subscriber may be changed by such a change. In addition, it is necessary to avoid the factor that hinders the service quality of the mobile communication subscriber in this process.

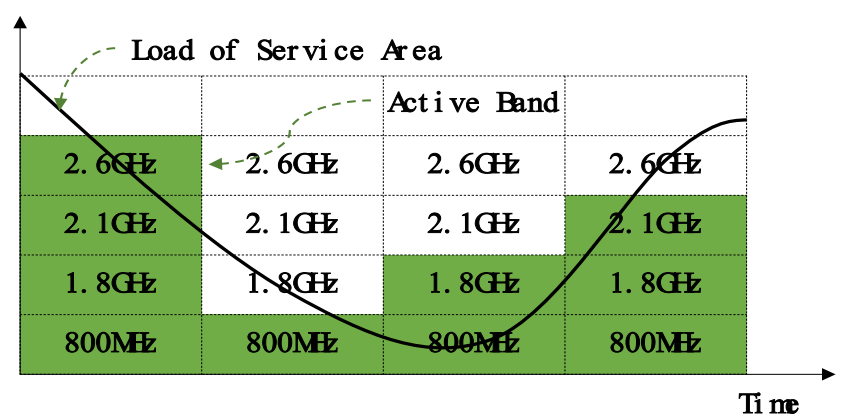

\section{PSi \& PSo Algorithm}

It is an ideal point of execution of power saving to minimize power consumption without affecting service quality of mobile subscriber. If you do not mind this ideal goal and run power saving, it is very likely that you will be shifting to negative side. When the service quality of the mobile communication subscriber is considered, the power saving level is lowered. On the contrary, if the power saving level is increased, the service quality of the subscriber is lowered. This means that when running power saving, you should not have to break down either your service quality or your power consumption. In this study, we have developed an algorithm that derives the optimal value between service level and power saving through three step approach.

(1) Power saving operation control variable regulation

(2) Subscriber service impact index calculation

(3) PSi \& PSo algorithm exploration

In order to find a clear power saving algorithm, we first define the control variables that can control the operation and clarify the input variables that the operator can set and control the algorithm. Then, in the step of measuring the change of the result, it is necessary to find an index capable of quantitatively judging the influence of the service of the mobile communication subscriber. After excavation, the amount of change must be analyzed through tracking and observation. After the analysis, we set the design direction of the algorithm by considering the determinants of the service impact. Finally, it is necessary to find a point that can maximize the power saving level while ensuring service quality for mobile phone subscribers. The PSi \& PSo algorithm established and stipulated these step - by - step core requirements and repeated the process of setting and analyzing each variable and outcome indicator.

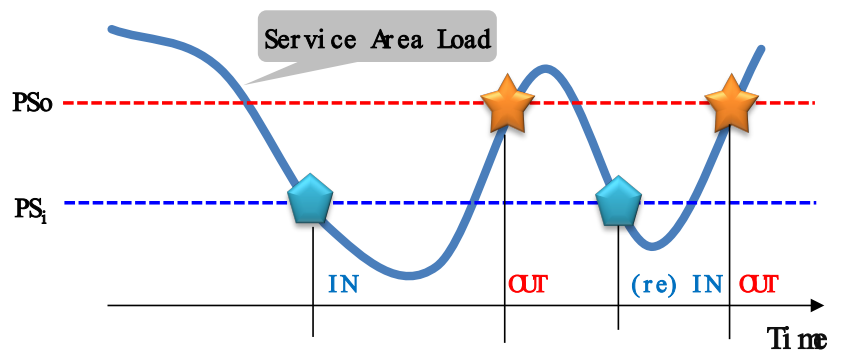

Figure 3: Power saving operation control variable 


\section{J. Kim et al. / Advances in Science, Technology and Engineering Systems Journal Vol. 4, No. 4, 47-50 (2019)}

\subsection{Control variables related to power saving operation}

First, in order to control the power saving operation, PSi, which is the power saving operation start step and PSo, which is the step of canceling the power saving operation, are defined based on the total load value of the service area.

As shown in Figure 3, the load on the service area changes over time. There is no difference in the fact that there is a difference in the number of times the load increases and decreases in each service area, but changes with time. When the load of the service area falls below a certain level in consideration of this variability, the power saving mode is entered (IN), and the value of this moment is defined as PSi. Conversely, when the load of the service area rises to a certain level or more, power saving mode is released (OUT), and the value of this moment is defined as PSo. When the power saving mode is entered (IN), the equipment of the specific frequency is turned off, and the remaining frequencies that are not off are able to handle the traffic of the subscriber, and power consumption is reduced from this moment. When the subscriber and traffic increase and the load is increased at the moment, it is necessary to turn on the frequency that was turned off to secure the proper capacity. Is turned on. From this moment on, power consumption is not reduced. Two variables, PSi and PSo, allow specific frequencies to be turned on or off to accommodate changing traffic in the service area. However, frequent entry (IN) and release (OUT) to the power saving mode may occur depending on changing traffic and load, which negatively affects the service quality of the mobile communication subscriber. In this paper, we try to find a way to maximize the reduction of power consumption through power saving operation and to minimize the influence of service quality of subscribers in the process.

\subsection{Definition of subscriber service impact indicators}

Although there are various indicators for calculating the service quality in the mobile communication network, it is necessary to adopt the connection rate $(\%)$ and the truncation rate (\%) that can comprehensively judge the quality of voice and data service. A new index CEI (Customer Experience Index) is calculated by multiplying two indicators and calculated by the following formula.

$$
\begin{aligned}
& \text { Call Connection Rate }(\%) \\
& \qquad=\frac{\text { Number of Connection Successful }}{\text { Number of Connection Attempt }} \times 100
\end{aligned}
$$

Call Drop Rate(\%)

$$
=\frac{\text { Number of Call Drop }}{\text { Number of Connection Successful }} \times 100
$$

$$
\text { CEI }(\%)=\text { Call Connection Rate } \times
$$$$
\text { (100 - Call Drop Rate) }
$$

\section{Equation 1: Estimation of the calculated index}

Let's look at Equation 1. As shown (1), it is possible to determine the success rate when the mobile communication terminal attempts to connect to the wireless network for the first time, and the truncation rate can be determined based on the connection rate of the mobile communication subscriber. It is possible to judge the situation in which the connection is released irrespective of the will of the user. (1) and (2), the environment of the service to be connected to the first wireless network, and the maintenance level of the connected state after the connection, and calculates the instant when the mobile communication subscriber attempts to connect to the network through the CEI of (3). It can be used as an indicator to represent the quality from the moment to the end.

\subsection{PSi \& PSo algorithm}

PSi and PSo, which are the control variables of power saving, are classified into three phases (high, mid, and lower) and the conditions for each stage are defined as follows.

Table 2: Understanding of PS control variable condition

\begin{tabular}{|c|c|c|}
\hline Division & Step & Condition \\
\hline PSi & High & Difficult to Entry \\
\cline { 2 - 3 } (Entry & Mid & Normal to Entry \\
\cline { 2 - 3 } Variable) & Low & Easy to Entry \\
\hline PSo & High & Easy to Release \\
\cline { 2 - 3 } (Release & Mid & Normal to Release \\
\cline { 2 - 3 } Variable) & Low & Difficult to Release \\
\cline { 2 - 3 }
\end{tabular}

Combining the three stages of PSi and the three stages of PSo, it can be combined the following nine cases. PSi \& PSo value means integrated load of one site.

Table 3: Test Case by PS Control

\begin{tabular}{|c|c|c|c|}
\hline Division & PSo(High): $\mathbf{3 0}$ & PSo(Mid): $\mathbf{4 0}$ & PSo(Low) : 50 \\
\hline PSi(High): 20 & Case1 & Case2 & Case 3 \\
\hline PSi(Mid): 30 & Case4 & Case 5 & Case6 \\
\hline PSi(Low): 40 & Case 7 & Case 8 & Case9 \\
\hline
\end{tabular}

Each case was tested in the commercial network of Namwon and Jangsu in Korea, which provides CA service of SK Telecom. The results of changing the PSi and PSo are summarized by the above CEI and the time when the equipment is off and not operated, and the cumulative calculated power saving value is added. Also, the values of PSi \& PSo set in each case are arbitrarily set, and they are set to a wide range in order to derive the optimal value.

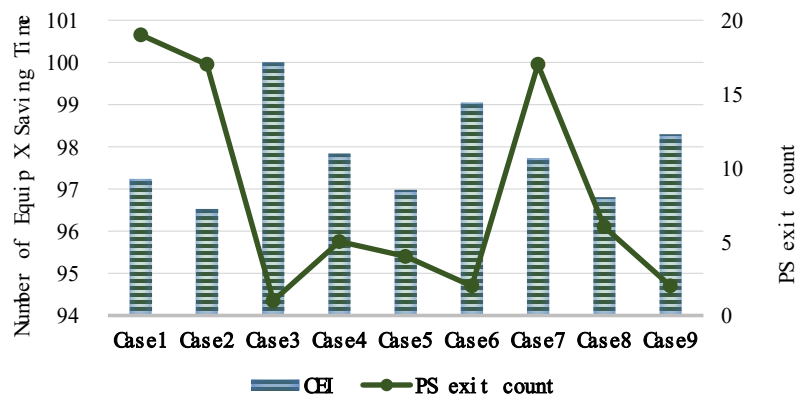

Figure 4 : Power saving exits and CEI by case

As shown in Figure 4, it can be seen that the CEI value varies depending on the case under test. And it can be clearly seen that it is inversely proportional to the number of times the power saving 


\section{J. Kim et al. / Advances in Science, Technology and Engineering Systems Journal Vol. 4, No. 4, 47-50 (2019)}

mode is abandoned. In other words, the more frequent the entry and exit into the power saving mode, the more negative the service quality of the subscribers. Conversely, the lower the number of exits (the longer stay in power saving Mode), the less negative impact on the service quality of the subscriber can be interpreted. Case \# 3, 6, and 9 had the fewest number of exits, and the service quality of the subscriber was maximized. As a result, it can be seen that adjusting the PSo to make it difficult to exit in power saving mode and reducing the number of exits has contributed to the optimum result preserving the CEI value.

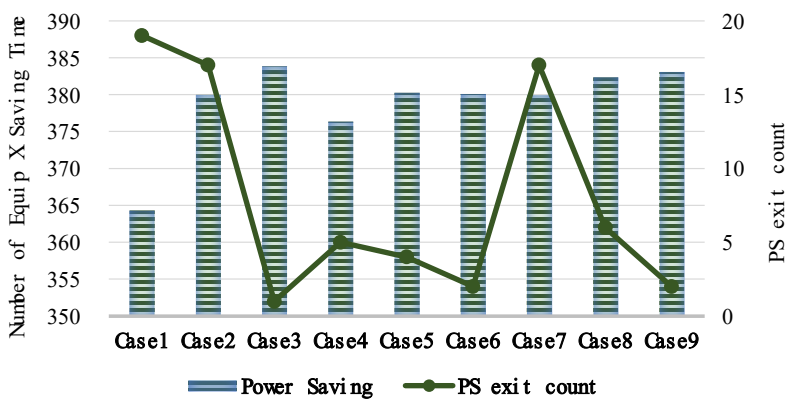

Figure 5 : Power saving level according to the number of power saving mode exits per case

The power saving level can be interpreted as PSi, which determines the entry, rather than the PSo condition, which interferes with the mobile communication subscriber experience when interpreting Figure 4 and Figure 5. In Case \# 1, 2, and 3, which are difficult to enter, the power saving level is low and the condition of entry is high in Case \# 7,8, and 9.

\subsection{Expanded application of PSi \& PSo algorithm}

The optimal values of PSi and PSo were selected as 20 and 50 respectively in SK Telecom. NOKIA developed a function to monitor the power consumed by mobile communication service equipment in real time. As a result of using this function, it is possible to save $0.12 \mathrm{~kW}$ when 1 hour off of $2.6 \mathrm{G}$ equipment. We extended it to the commercial network in 2019 and saved about $\$$ 93,000 over five months. Also, it is confirmed that CEI, which is an indicator of service quality, is maintained within the normal range.

As mentioned above, the lower the number of exits to the power saving mode, the lower the service quality of the subscriber can be prevented. As a result of applying power saving to $2.6 \mathrm{G}$ equipment in Jeju region of Korea, we found a device with maximum mode change count of 66 times. The site has a lot of tourists, and the traffic load is very volatile. There are two options for these areas. In order to maintain the service quality of the subscriber, it is necessary to abandon the power saving or set the PSi value low to make it difficult to enter the power saving mode (IN), thereby performing the power saving only for the necessary time. The author chose to give up power saving in the area. It is also important to reduce electricity consumption by reducing electricity consumption. However, it is because the company regards the service quality of its subscribers as more important. If one day the company's policy changes and minimizes operating costs become more important, it can turn into a situation where power savings can be applied to these areas and the service quality degradation of subscribers can be tolerated to some extent.
SK Telecom will continue the power saving in order to reduce the electricity cost to operate the network. However, it considers the quality preservation of service subscribers as the top priority and repeats the process of continuously checking the PSi \& PSo algorithm and deriving the optimum value will be.

\section{Conclusion}

The PSi and PSo algorithms for the power saving method that maximize the reduction of power consumption while maintaining the service quality of the mobile communication subscriber are discussed. In order to derive the results, we have divided into 9 cases and the optimal value of PSi and PSo in the area managed by the author can be derived as PSi $=20 \&$ PSo $=50$. This number will vary depending on the traffic conditions in each region served. We concluded that we can adjust the power saving mode deviation to minimize PSo and access the ideal result of power saving execution if the PSi-controlled entry operation is easily set up. Although this study investigated algorithms for power saving, we did not discuss the various ISSUEs that can occur when applying this algorithm to commercial networks and the values that should be prioritized by each company's policy. This is because it depends on the value and philosophy of the mobile service provider and operator adopting this algorithm. However, the load fluctuation differs according to the service area and the load level that can be tolerated during the power saving execution may be different between the mobile communication company executing the same and the organization within it. Thus, the task of identifying the optimal value for the demand level is left as an additional research area. In addition, this approach has a weak point that it can partially obstruct the service quality of the mobile communication subscriber during the process, but it has a strong point that it is possible to draw conclusions that can be immediately expanded through direct execution in the commercial network.

Based on the results of this study, the operators who are interested in the reduction of the electricity cost among the network operation cost will be able to execute immediately because they have obtained the initial value and the method that maximizes the power consumption reduction while minimizing the quality deterioration of the subscriber.

\section{References}

[1] Kim, Jongsin, Yonggil Choi, and Younghoon Oh. "The Study of PSi \& PSo Algorithm for Reducing Power of the Mobile Communication Network." 2018 International Conference on Information and Communication Technology Convergence (ICTC). IEEE, 2018.

[2] 3GPP TR 36.822, LTE Radio Access Network (RAN)enhancements for diverse data applications (Release 11)

[3] Korea Communication Commission, Jung Woo-su, Kim Seung-gun. A study on "Research on the ripple effects of telecom operators' investment activities". Research on broadcasting communication policy, A Reserch report.

[4] Hyun suk-bong, Kang sung-won, Um nak-woong. Low Power SoC Technology Wireless Terminals. Electronics and telecommunications trends, Vol.23 No.6

[5] Kim tae-yong, Cho gyu-seong, Jo sung-ho. A Study on CDRX operation of eNB for battery saving in LTE smartphone. The Journal of Korean Institute of Communications and Information Sciences.

[6] Aderemi A. AtayeroOluwadamilola I. AduAdeyemi A. Alatishe, "Self Organizing Networks for 3GPP LTE" 